\title{
Study on Knowledge Sharing of Community of Practice Based on Social Network Perspective
}

\author{
Kaiji Liao, Huihui Xiong \\ School of Business Administration, South China University of Technology, Guangzhou, China. \\ Email: xhh352@126.com
}

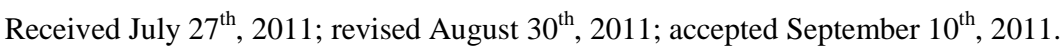

\begin{abstract}
Based on social network perspective, we developed a model about the knowledge sharing of communities of practice (CoPs), analyzed the knowledge sharing of CoPs from the view of the network density, network centrality, network structure. And we found out that only the above indexes are kept in appropriately level can stimulate the knowledge sharing in CoPs. The strong ties contribute to the transfer of implicit knowledge, while the weak ties promote explicit knowledge. At last, according to the result of our study, we proposed some suggestions for further study on knowledge sharing of CoPs.
\end{abstract}

Keywords: Social Network Analysis, Community of Practice, Knowledge Sharing

\section{Introduction}

Communities of practice as an informal organization which is different from the formal organization, has an important influence on improving organization's performance. Then how to ensure knowledge sharing of communities of practice becomes a key problem. Previous studies on communities of practice are from the perspective of the qualitative analysis of organizational structure, very little quantitative analysis from the perspective of research. Social network analysis belongs to the scope of sociology, and has been applied to knowledge sharing in some progress, but when it applied to communities of practice, the related have not been studied. Social network analysis can be a useful quantitative analytical tool in studying community of practice on the dissemination of knowledge.

\section{Community of Practice}

Wenger [1] defined communities of practice as "groups of people who share a concern or a passion for something they do and learn how to do it better as they interact regularly". According to Wenger's definition, communities of practice must has the following three features: the domain, that communities of practice must be related to one or some areas; the members through participation in community activities, share their experiences, and learn from each other; the practice, the members according to their areas of interest to exchange and to maintain sustained interaction. The community of practice is spontaneous action of the members of areas, therefore, has the informal nature. And the informal organization plays an important role in promoting the sharing of tacit knowledge, and help tacit knowledge externalized. However, existing studies are mainly from the perspective of qualitative analysis to assess community of practice, little quantitative research methods.

\section{Social Network Analysis}

Social network analysis describes the relationship between people, groups, organizations, computers, or other information, knowledge processing entities, and evaluates its values [2]. Network nodes indicate subject, the connections between nodes represent the main link. Social network analysis can be mapped to the social relationship network between members of CoPs, and make knowledge sharing in CoPs more clearly. What's more important, social network analysis is different from the past qualitative study. This paper developed a quantitative research method to advance the knowledge sharing in CoPs. The result shows that it can help companies achieving strategic goals. In this paper, we analyzed the behavior of knowledge sharing in CoPs both from the overall structure of the network and the strength of connections. 


\subsection{The Overall Structure of the Social Network}

1) Network density: shows the degree of connection between the nodes of social network, which can reflects the density of relationship in social networks. For simple undirected network, the network density which can be represented by the ratio of actual number of connections and the maximum total number of connections that may exist. Such as Equation (1).

$$
\text { density }=2 l / n(n-1)
$$

where $l$ is the actual number of network connections, $n$ is the number of network nodes [3].

2) Centrality: shows status and influence of nodes in the network. For a network, it's centrality can be obtained from the following method: first to identify the maximum degree $C_{\max }$ in figure; then calculate the value diminish the degree of centrality of any other nodes $C_{\max }-C_{i}$, so we get a lot of "difference value"; again calculate the sum of these "difference value"; at last, divides the value into the maximum possible value of those difference values, see in formula (2).

$$
\text { centralization }=\frac{\sum_{i=1}^{n}\left(C_{\max }-C_{i}\right)}{\max \left[\sum_{i=1}^{n} C_{\max }-C_{i}\right]}
$$

which $i$ represent any of the nodes, $n$ represents the number of network nodes.

3) The form of the overall structure of the network: there are two categories: small group structure and core periphery structure [4] which is shown in Figure 1 and Figure 2. The small group structure is made up of a number of close ties within small groups which link through the bridge. Core-periphery structure is characterized by the existence of a close connection within the core group, while the other members are mainly linked with the core members, other members have less direct contact with each other.

\subsection{The Strength of the Social Relationships}

Granovetter in his article "strength of weak ties" said that the connection strength between network members can be measured by "time we spent, emotional commitment, intimacy and reciprocal services on a link". Strong ties are those frequent, persistent, passionate relations; the weak ties are those occasional, distant relations [5]. As shown in Figure 3.

\section{A Model of the Knowledge-Sharing Communities of Practice Based on Social Network Analysis Perspective}

The most important feature of CoPs is the informal net-

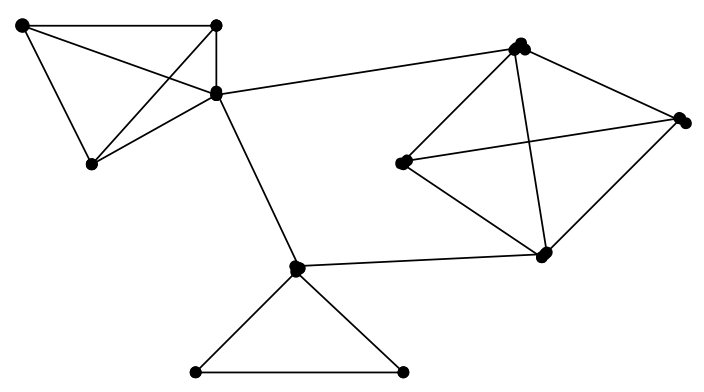

Figure 1. Small group structure.

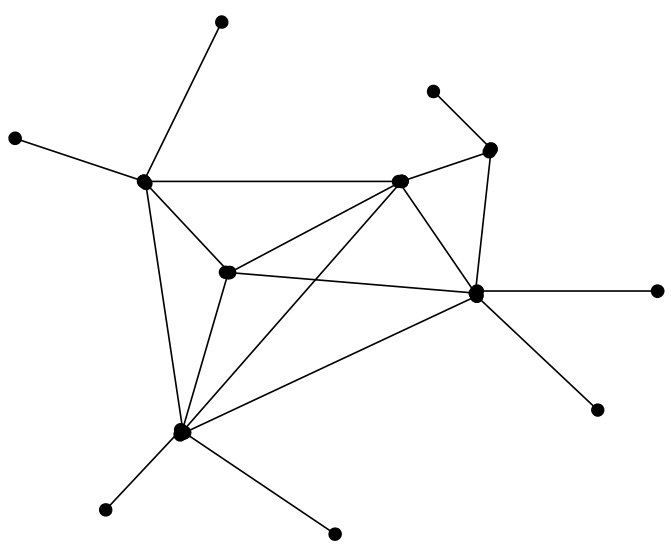

Figure 2. Core-periphery structure.

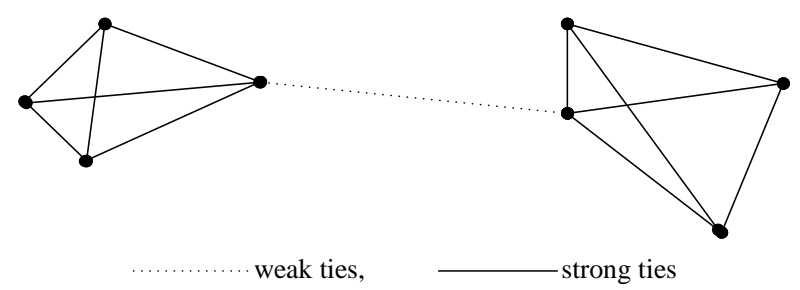

Figure 3. Strong ties and weak ties.

work, with self-organization. Community members keep continuous interaction through network or face to face communication, and achieve the purpose of sharing knowledge. The main practice in the community is tacit knowledge sharing, and the method of social network analysis can be mapped to the social relationship network of CoPs among members of the community, so knowledge-sharing in communities of practice becomes more clearly. What's more important, social network analysis is different from those qualitative research methods in the past, it is a quantitative research method which promoting knowledge sharing in communities of practice.

We developed a model of knowledge sharing of CoPs based on social network analysis, shown in Figure 4. The model research into knowledge sharing in CoPs is mainly from the four parts of social network, such as network density, centrality, small groups, the core--pe- 


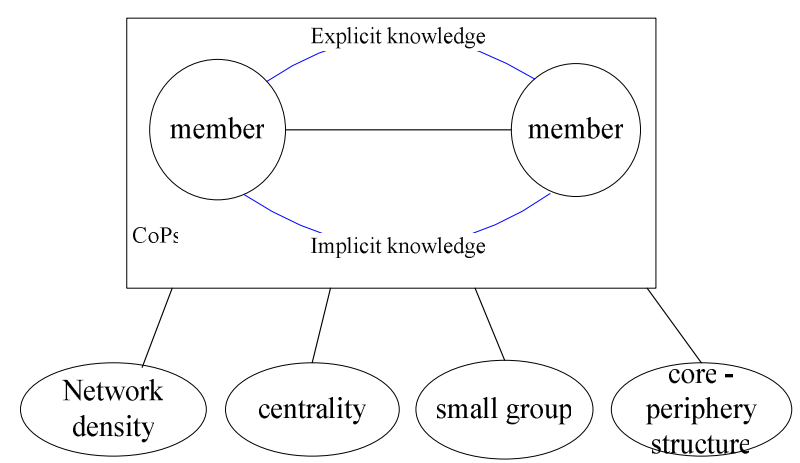

Figure 4. A model of knowledge sharing of communities of practice based on social network analysis.

riphery structure, and relations between network members.

\subsection{The Overall Structure of the Network in Model}

1) Network density

If the network density in communities of practice is too low, the relationship among members is relatively weak. It is obviously not conducive to knowledge sharing behavior; in the other hand, the higher density means that will have the opposite effect on the performance of community. At present, a number of empirical studies show that too high or low density of the network are not conducive to the sharing of knowledge dissemination, so the network density required to maintain at an appropriate level [6,7].

2) Centrality

Network centrality indicates the centralization degree of network, which focused on the situation of the minority interactive human in communities of practice. Network centrality has a negative correlation with organizational performance [8]. The higher network centrality means that power is concentrated in few hands, while the mutual exchanges between other members are less. Once the central figure leaves communities of practice, accompany with a greater loss on knowledge sharing. However, the lower centrality of communities of practice indicates that community interaction is over-decentralized; therefore the ability of creation of membership will be diminished [9]. To sum up, the centrality of the network is too high or too low is not conducive to knowledge-sharing communities of practice, but to maintain at an adequate level.

3) The overall structure of network

Since the overall structure of the network usually with those two forms: small groups or core-periphery structure. For the structural holes of the small group structure is the non-repetition relationship between small groups $[10,11]$. The existence of structural holes in network will become the bottleneck impeding the flow of information in CoPs. The corresponding bridge is the weak link between small groups, through which small groups can be linked with each other and can communicate information, and will have different information and knowledge [12]. Thus established a bridge which links individuals which have different knowledge, it contributes to the knowledge sharing.

For the core-periphery structure, as the core members of the network have close connection with other members, but the edge members little association with members.

\subsection{The Strength of the Relationships in Model}

Strong ties can do favor of knowledge sharing and transfer of knowledge in communities of practice particularly the tacit knowledge; while weak ties have dominant place in expanding the scope of transfer of knowledge sharing in the community, and accessing to heterogeneous resources [13]. That's because the knowledge that shared in communities of practice is mainly tacit knowledge, and tacit knowledge itself has the characteristics that is tacit and difficult to transfer.

A successful knowledge transfer often requires members of the two sides have repeatedly cooperation. The strong ties mean that close relations, and easy to cultivate trust among the members. At the same time, strong ties help restraint the threat of opportunism of tacit knowledge sharing, and remove barriers of knowledge sharing. Therefore, compared to weak ties, strong ties are more likely to promote the sharing of tacit knowledge [14,15].

The weak ties members do not contact each other very frequently, and their relations are indifferent. But the weak ties can cross domain members. According to Granovetter's point of view, it is easy to get new heterogeneous information through the weak ties, more and more members can be connected by weak ties which are conducive to expanding the scope of knowledge transfer, particularly the transfer of explicit knowledge.

\section{Conclusions}

The communities of practices are different with formal organization, while past studies on communities of practice are mainly qualitative analysis, there is little quantitative research. In this paper, we developed a model about the knowledge sharing of communities of practice (CoPs) based on the social network perspective, analyzed the knowledge sharing of CoPs from the network density, network centrality, network structure. And found that the strong ties contribute to the transfer of implicit knowledge, while the weak ties promote explicit knowledge. The relationships and the structure of network must be kept in appropriately level can stimulate the knowledge 
sharing in CoPs. Further studies of specific communities of practice can be made in the future, we can choose a specific community to conduct empirical research, by collecting data, processing them and obtaining various specific targets by using Ucinet software.

\section{REFERENCES}

[1] E. Wenger, “Communities of Practice,” 2006. http://www.ewenger.com/theory/index.htm

[2] V. Krebs, "Social Network Analysis," 2005. http://www.orgnet.com/sna.html

[3] J. Liu, "Introduction to Social Network Analysis," Social Sciences Academic Press, Beijing, 2004.

[4] J. N. Cummings and R. Cross, "Structural Properties of Work Groups and Their Consequence for Performance," Social Network, Vol. 25, No. 3, 2003, pp. 197-210.

[5] M. S. Granovetter, “The Strength of Weak Ties,” American Journal of Sociology, Vol. 78, No. 6, 1973, pp. 13601380.

[6] M. Thompson, "Structural and Epistemic Parameters in Communities of Practice," Organization Science, Vol. 16, No. 2, 2005, pp.151-164. doi:10.1287/orsc.1050.0120

[7] J. D. Luo, "Social Network Analysis," Social Science Academic Press, Beijing, 2005.

[8] M. E. Shaw, “Communication Networks,” In: L. Berkowitz Ed., Advances in Experimental Social Psychology,
Academic Press, New York, 1964, pp. 111-147.

[9] H. Ibarra, "Network Centrality, Power, and Innovation Involvement: Determinants of Technical and Administrative Roles," Academy of Management Journal, Vol. 36, No. 3, 1993, pp. 471-501. doi:10.2307/256589

[10] R. Burt, "Structural Holes: The Social Structure of Competition," Harvard University Press, Cambridge, 1992.

[11] M. Hansen. "The Search-Transfer Problem: The Role of Weak Tie in Sharing Knowledge across Organization Subunits,” Administrative Science Quarterly, Vol. 44, No. 1, 1999, pp. 82-111. doi:10.2307/2667032

[12] J. N. Cummings and R. Cross, "Structural Properties of Work Groups and Their Consequences for Performance," Social Networks, Vol. 25, No. 3, 2003, pp. 197-210. doi:10.1016/S0378-8733(02)00049-7

[13] C. Carey, K. Smith and L. M. Martin, “Cross-University Enterprise Education Collaboration as a Community of Practice,” Education and Trainning, Vol. 51 No. 8-9, 2009, pp.696-706.

[14] N.-T. Huang, C.-C. Wei and W.-K. Chang, "Knowledge Management: Modeling the Knowledge Diffusion in Community of Practice," Kybernetes, Vol. 36, No. 5-6, 2007, pp. 607-621.

[15] G. P. Yin, Y. S. Mo and Y. Chen, "Promoting Tacit Knowledge Management Using Social Network Analysis," Journal of Tsinghua University (Science and Technology), Vol. 46, No. S1, 2006, pp. 964-969. 